\title{
DYNAMIC OPTIMITATION IN THE GAIT ANALYSIS IN NORMAL AND PATHOLOGICAL SITUATIONS
}

\author{
V. Crupi, G. La Rosa and R. Sinatra \\ Istituto di Macchine, Facoltà di Ingegneria, \\ Università di Catania, \\ Viale A. Doria 6, 95125 Catania, Italy \\ vcrupi@im.ing.unict.it, glarosa@im.ing.unict.it, rsinatra@im.ing.unict.it
}

\section{INTRODUCTION}

The main advantage of the modern fixation implants, respect to the traditional plastering, lies in allowing the immediate walking to the patient. The direct application of mechanical loads was noticed to promote the callous growth and to reduce the recovery time. The greater forces involved must be carefully considered during the design of the prostheses or the fixation systems in order to assure the stability of the bone stumps in the fracture zone. Being the bones as composite materials, not only the intensity of the forces has to be evaluated, but their directions too; the main problems arise with the sharp application of the load (high strain rate) and in a direction different from the physiological one.

One of the purpose of the simulation carried out is the determination of the space-time state for the forces and the moments in the articulating joints and the definition of the contact forces. The results of the simulation were compared with the values found in literature, obtained in. experimental tests using force platforms. Many static models were developed in the past, considering the human body as a kinematic chain consisting in rigid bodies linked by revolute joints. The intersegmental forces, representing the articulating forces were calculated applying the equations of equilibrium for every rigid body, neglecting the inertial forces. The external forces, then, the body weight and the ground reaction, were supposed equal in intensity and opposite in direction; but it is not true if we consider the inertial force. Only using a dynamic multi-body software, the whole force state can be considered.

\section{METHODS}

The numerical simulation was performed by the software MDI/ADAMS on workstation. In the numerical modelling the human body was considered as a multi-body system by 15 rigid parts linked by 14 revolute joints. The simulation of the normal gait was carried out using the data found in literature, in order to assure the reliability of the model. To calibrate the pathological gait, the walking of patients after 60 days from the surgical operation of hip arthrosis was studied by an image analysis system, applying several light markers on the patient skin in correspondence of the articulating joints. By the system experimental data were acquired on the most important kinematic parameters (speed, gait time, gait length, etc.) and the relative angles during a step. Both the limbs were considered, being the pathological gait non symmetric. All the values of the angles for all the articulating joints were used as input in the numerical model. 
The resultant joint force and joint moment, obtained using the inverse dynamics approach, were the sum of muscular, ligamentous and bone contact forces. The unconstrained N-E equations are a mathematically undetermined system. An optimation programme was used to solve the system of equations. Different forms of objective function were considered: muscolar forces, muscolar stress, muscolar work, etc. The numerical results were compared. The aim of this numerical simulation is to obtain the muscular and bone contact force, wich act on the bone during a single gait.

\section{RESULTS}

The android, as in the real gait, leans the foot with the heel, when in the push phase he uses the tiptoe and the step is symmetric for both the legs. According to the values found in literature, the contact phase is long about the $60 \%$ of the total step time and the jump phase the remaining $40 \%$. The values of the angles were calculated for the whole step and the kinematic parameters were compared with those found in the literature and in the experimental tests for the normal gait. By the experimental analysis a large difference between the normal and pathological step was noticed, corresponding to the numerical results on the numerical analysis. Based on the results, an integrated set of mechanical parameters (time, forces, moments, energy, reactions, etc.) was calculated to have a better knowledge of the load state in pathological situations. In the figures bottom are reported the horizontal and vertical components of ground reaction force during the stance phase of a single gait. The numerical values were calculated for phisiological and pathologiacal situations. In the pathological case the gait is not symmetric for the two legs (intact and sore), so the forces values for both legs were considered. 


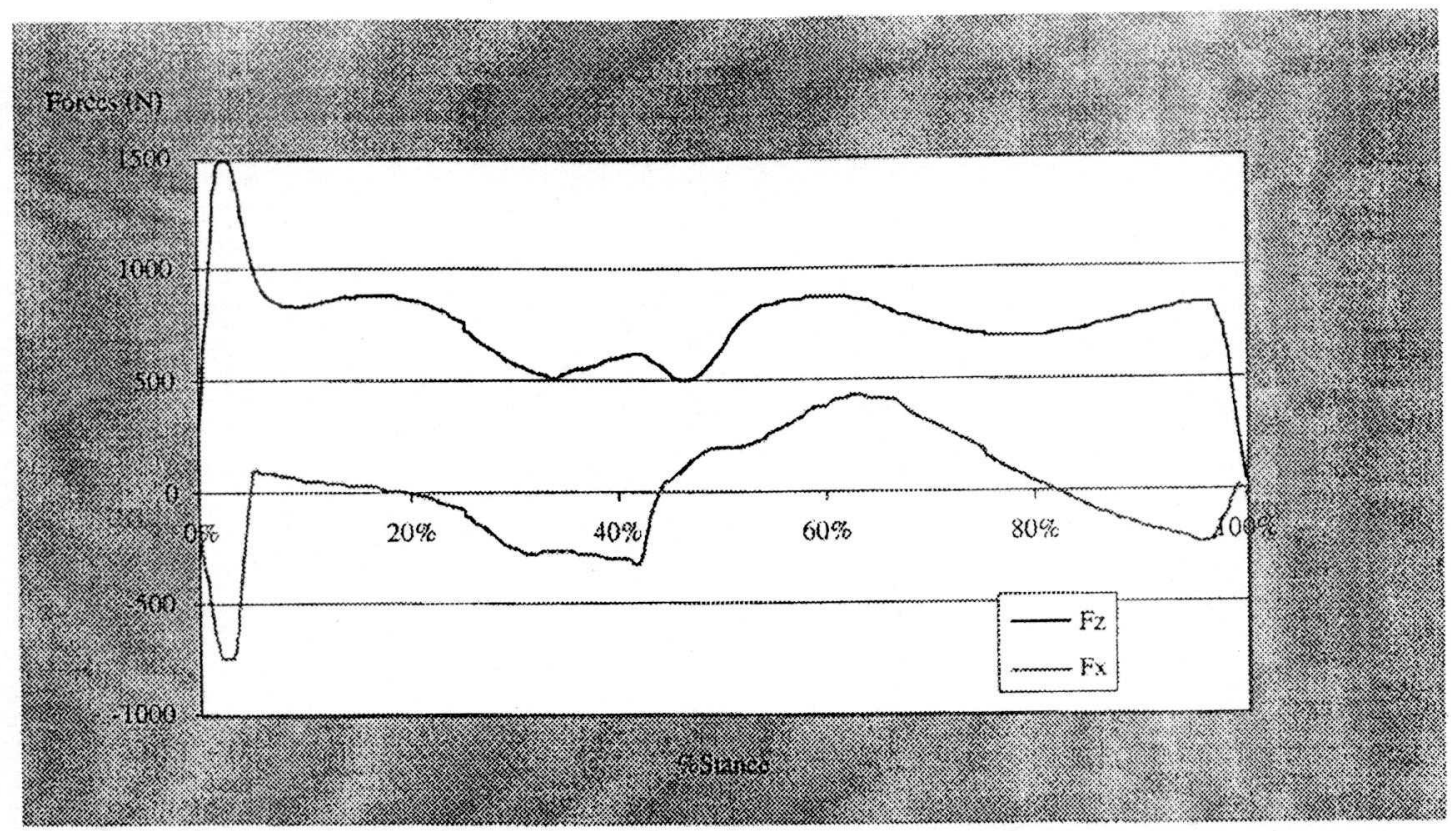

Figure 1: ground reaction force components during the stance gait phase of phisiological situation.

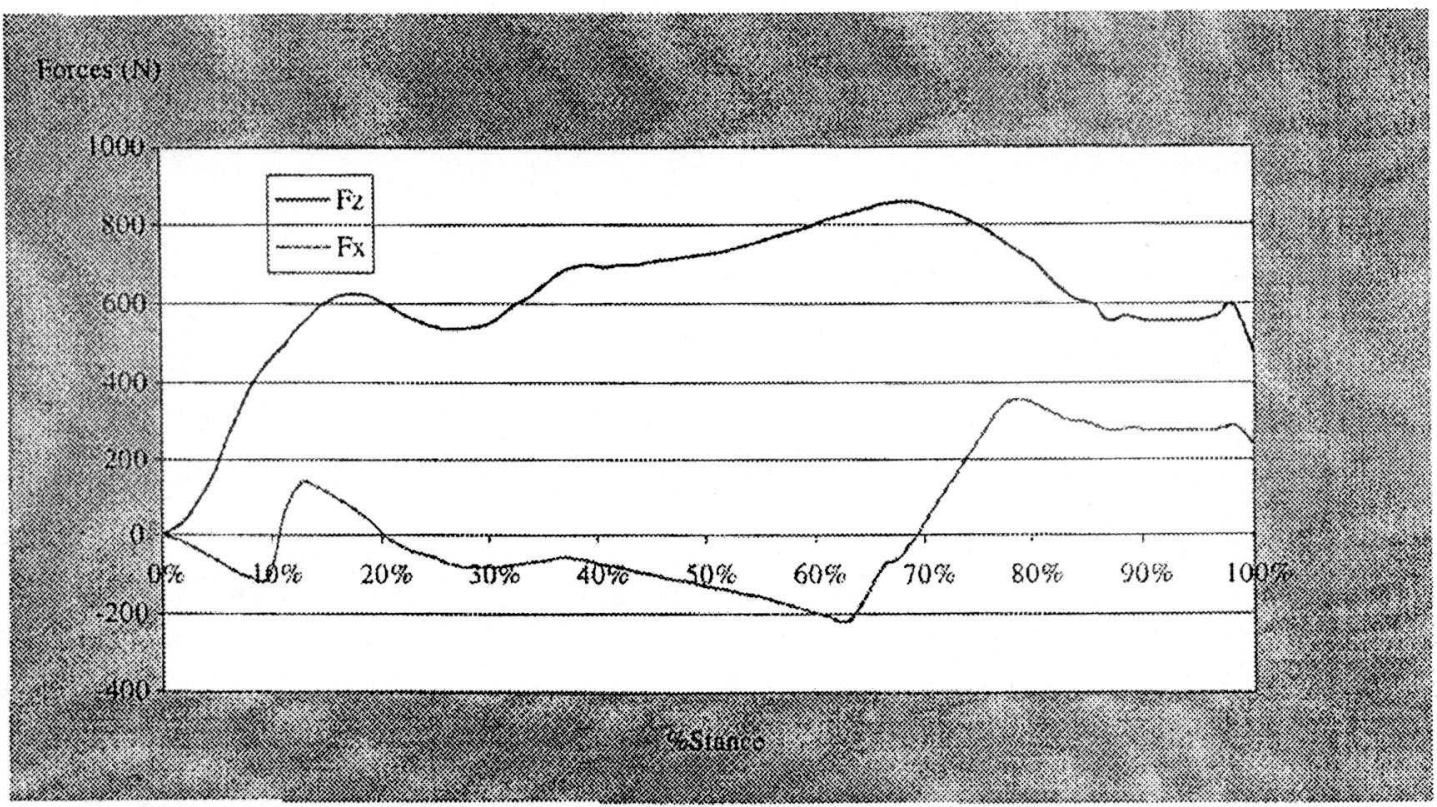

Figure 2: ground reaction force components during the stance gait phase of phathological situation for sore leg. 


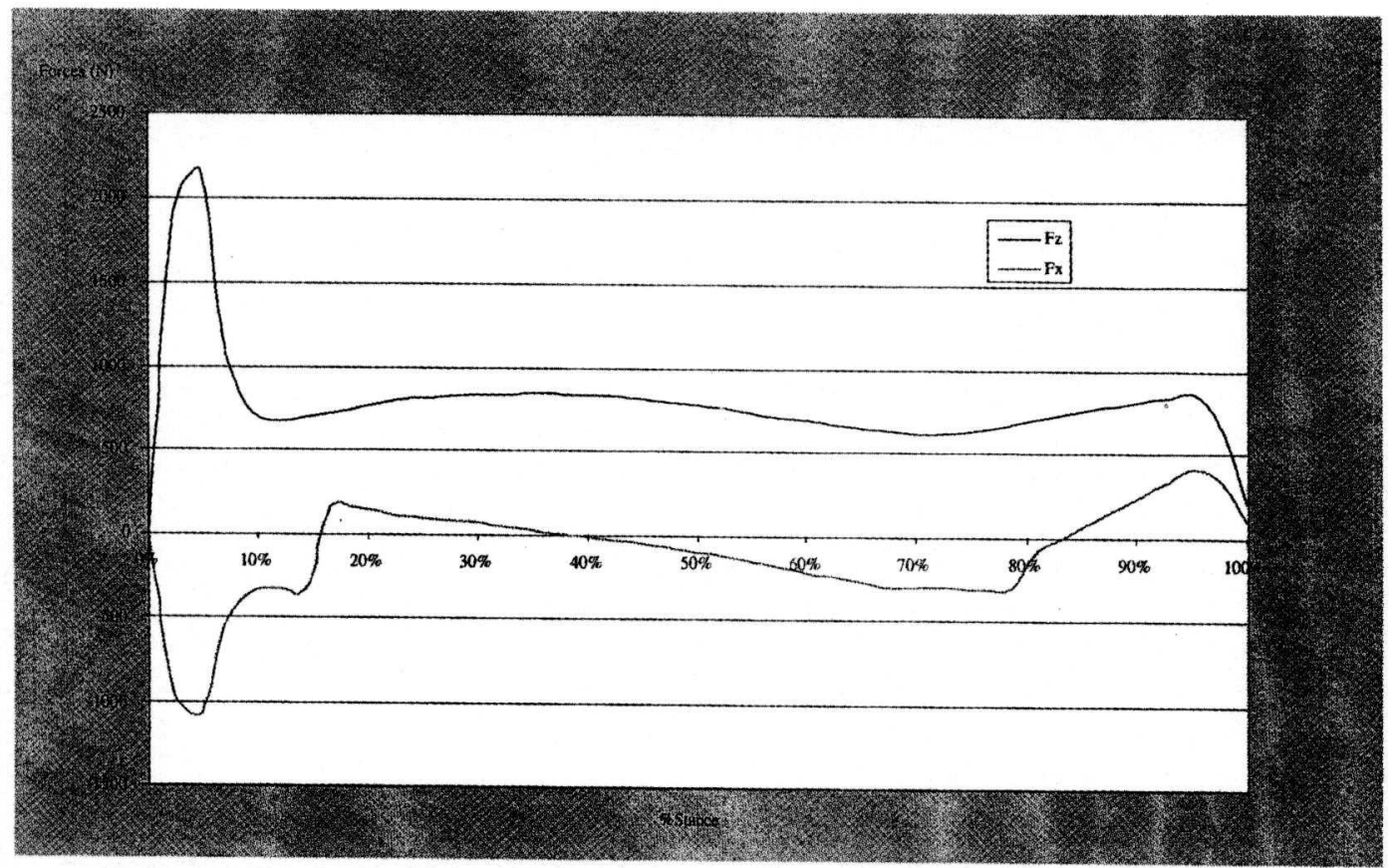

Figure 3: ground reaction force components during the stance gait phase of phathological situation for intact leg.

\section{REFERENCES}

1. Chou L.S., Song S.M., Draganich: Predicting the kinematics and kinetics of gait based on the optimum trajectory of the swing limb, Journal of Biomechanics, Vol.28, pp. $377-385,1995$.

2. Beckett R., Chang K., An evaluation of kinematics of gait by minimum energy, Journal of Biomechanics, Vol.1, pp. 147 - 159, 1996.

3. Duda G. N., Schneider E., Chao Y. S.: Internal forces and moment in the femour during walking, Journal of Biomechanics, Vol. 30, pp 933-941, 1997.

4. Pandy M. G., Berme N.: Quantitative assestment of gait determinants during single stance via a three dimensional model, Journal of Biomechanics, Vol. 22, pp 717-733, 1989.

5. Zarrugh M.Y.: Kinematic prediction of intersegment loads and power at the joint of the leg in walking, Journal of Biomechanics, Vol. 14, pp 713-725, 1981.

6. Onyshko S., Winter D.A.: A mathematical model for the dynamics of human locomotion, Journal of Biomechanics, Vol. 13, pp $361-368,1979$. 